\title{
EDITORIAL
}

\section{Exposure science in the 21 st century: a vision and a strategy}

Journal of Exposure Science and Environmental Epidemiology (2013) 23, 1; doi:10.1038/jes.2012.109

A Report of the National Research Council (NRC) - September 2012: in response to a request from the Environmental Protection Agency (EPA) and the National Institute of Environmental Health Sciences (NIEHS).

The report was prepared by the NRC Committee on Human and Environmental Exposure Science in the 21st Century (Kirk R Smith, Chair; Paul J Lioy, Vice Chair). It provides: (1) an authoritative review of the current state of environmental exposure science; (2) snapshots of opportunities that have been created in recent years in the broader scientific field that can be harnessed to create new tools for advances in the scope and practice of exposure science, especially in miniaturization of highly sensitive pollutant detectors and biomarkers, the management of databases, and advances in information technology; and (3) discussions of some of the impediments that need to be overcome in implementing a strategy to match the Committee's vision.

The vision includes a broader recognition of the need for more capable methods for exposure assessments that can serve to support: (1) more credible environmental and health risk assessments; (2) more quantitative epidemiological studies of environmental and human exposure - response relationships; and (3) more thorough evaluation of exposure-response relationships in toxicological studies, including exposures to internal targets (e.g. critical organs and cells), and applications of biomarkers of exposures and responses.

The impediments include: (1) insufficient funds and funding mechanisms that can support enough research to develop new measurement tools and models that will be needed, both within the EPA and NIEHS laboratories, and in the university and other non-profit research centers; (2) gaining greater access to more governmental and proprietary databases to support model development and validation; and (3) insufficient recognition by colleagues engaged in toxicology, epidemiology, and risk assessment of how they can benefit from more collaboration with exposure science practitioners.

The readers of this journal will need to download the new NRC Report, digest its findings, and begin to engage in outreach and more dialog with epidemiologists, toxicologists, and risk assessors, which will lead to more collaborative endeavors that will advance our collective contributions to public health and environmental protection and, at the same time, advance the recognition of exposure science as a critical discipline and contributor to the overall endeavor.

\author{
Morton Lippmann ${ }^{1}$ \\ Editor-in-Chief, JESEE \\ ${ }^{1}$ Department of Environmental Medicine, NYU Langone Medical \\ Center, Sterling Forest, Tuxedo, NY 10987, USA \\ E-mail: morton.lippmann@nyumc.org
}

